\title{
THE DIRECT DECOMPOSITION OF $l$-ALGEBRAS INTO PRODUCTS OF SUBDIRECTLY IRREDUCIBLE FACTORS
}

\author{
SÁNDOR RADELECZKI
}

(Received 28 March 2001; revised 1 July 2002)

Communicated by B. A. Davey

\begin{abstract}
Generalizing earlier results of Katriňák, El-Assar and the present author we prove new structure theorems for $l$-algebras. We obtain necessary and sufficient conditions for the decomposition of an arbitrary bounded lattice into a direct product of (finitely) subdirectly irreducible lattices.
\end{abstract}

2000 Mathematics subject classification: primary 08A05, 06F99, 06B05.

\section{Introduction}

It is well known that geometric lattices are direct products of subdirectly irreducible geometric lattices. This result naturally involves the question: 'Under what conditions a lattice $L$ can be decomposed into a direct product of subdirectly irreducible lattices?' In [11] the author of this paper proved:

THEOREM 1.1. Let $L$ be a $C J$-generated algebraic lattice. Then the following are equivalent:

(i) $L$ is a direct product of subdirectly irreducible lattices.

(ii) $L$ enjoys property (PCC) and $\operatorname{Con} L$ is an atomic Stone lattice.

We say that a congruence distributive algebra $\mathbf{A}$ enjoys property ( $\mathrm{PCC}$ ), if any complemented congruence of A permutes with its complement.

Katriňák and El-Assar investigated a similar problem [8], for congruence distributive algebras. One of their important results is the following:

Research partially supported by Hungarian National Foundation for Scientific Research (Grant No. T029525 and No. T034 137) and by János Bolyai Grant of Hungarian Academy of Science.

(C) 2003 Australian Mathematical Society 1446-7887/03 $\$$ A2.00+0.00 
THEOREM 1.2 ([8, Theorem 11 (iii)]). Let $\mathbf{A}$ be a congruence distributive algebra with a strong centre and enjoying property (PCC). Then Con $\mathbf{A}$ is (atomic and) completely Stonean if and only if $\mathbf{A}$ is a finite direct product of finitely subdirectly irreducible (subdirectly irreducible) algebras.

In [8] they applied their results to the class of so called $l$-algebras (see Definition 2.4 (iii)) too.

Comparing the above two results, it seems that Theorem 1.1 can be valid in a more general context. Our main result can be considered as a common generalization of Theorem 1.1 and Theorem 1.2 for $l$-algebras. This is the following:

THEOREM 1.3. Let $\mathscr{L}$ be an l-algebra. Then

(1) $\mathscr{L}$ is a direct product of finitely subdirectly irreducible l-algebras if and only if $\mathscr{L}$ enjoys property (PCC), Con $\mathscr{L}$ is a Stone lattice and the underlying lattice $L$ is weakly central-complete with an atomic center.

(2) $\mathscr{L}$ is a direct product of subdirectly irreducible l-algebras if and only if $\mathscr{L}$ enjoys property (PCC), Con $\mathscr{L}$ is an atomic Stone lattice and the underlying lattice $L$ is weakly central-complete.

Since any bounded lattice is a particular $l$-algebra, Theorem 3.1 can be also applied to bounded lattices.

The proof of this theorem can be found in Section 5. The preliminary notions and some technical results are contained in Section 2. In Section 3 we deal with product decompositions of congruence distributive algebras. The principal result of this section is Theorem 3.1, which will prove a useful tool in our development. In Section 4 we prove a necessary and sufficient condition for the decomposition of an arbitrary bounded lattice into a direct product of directly indecomposable lattices. In Sections 6 and 7 we apply Theorem 1.3 to certain classes of $l$-algebras and complete lattices.

\section{Preliminaries}

Let 0 and 1 stand for the least and the greatest element of a bounded lattice $L$. The principal ideal and the principal filter generated by an $x \in L$ will be denoted by $(x]$ and $[x)$, respectively. A bounded lattice $L$ is called atomic if for any $x \in L, x \neq 0$ the interval $[0, x]$ contains at least one atom of $L$.

DEFINITION 2.1. Let $L$ be a bounded lattice. An element $a \in L$ is called a central element of $L$ if $a$ is complemented and for all $x, y \in L$ the sublattice generated by $\{a, x, y\}$ is distributive. 
The central elements of a (bounded) lattice $L$ form a Boolean sublattice of $L$ denoted by $\operatorname{Cen}(L)$. A complement of an element $a \in L$ (if it exists) is denoted by $\bar{a}$. For any $c \in \operatorname{Cen}(L)$, we define the relation

$$
\theta_{c}=\{(x, y) \mid x \vee y=(x \wedge y) \vee a, \text { for some } a \leq c\}
$$

It is easy to check that $\theta_{c}$ is a congruence of $L$ and that

$$
(x, y) \in \theta_{c} \quad \text { if and only if } \quad x \wedge \bar{c}=y \wedge \bar{c} .
$$

REMARK 2.2. The following simple observations can be found, for instance, in [4]:

(i) For any $c_{1}, c_{2} \in \operatorname{Cen}(L)$, we have $\theta_{c_{1} \vee c_{2}}=\theta_{c_{1}} \vee \theta_{c_{2}}$ and $\theta_{c_{1} \wedge c_{2}}=\theta_{c_{1}} \wedge \theta_{c_{2}}$. If $c_{1} \leq c_{2}$ then $\theta_{c_{1}} \leq \theta_{c_{2}}$, and $\theta_{c_{1}}=\theta_{c_{2}}$ implies $c_{1}=c_{2}$.

(ii) For any $c \in \operatorname{Cen}(L), \theta_{c}$ and $\theta_{\bar{c}}$ form a factor congruence pair of $L$ and conversely, if $\theta_{1}$ and $\theta_{2}$ are factor congruences of a bounded lattice $L$, that is, $L \cong L / \theta_{1} \times L / \theta_{2}$, then there exists a $c \in \operatorname{Cen}(L)$ such that $\theta_{1}=\theta_{c}, \theta_{2}=\theta_{\bar{c}}$. Moreover $L / \theta_{\bar{c}} \cong(c]$.

The following assertion can be easily proved.

LEMMA 2.3. In any bounded lattice $L=\prod_{i \in I} L_{i}$ there exist elements $c_{i} \in \operatorname{Cen}(L)$, $i \in I$ such that $L / \theta_{\overline{c_{i}}} \cong L_{i}$.

Let $(S, \wedge, 0,1)$ denote a bounded meet-semilattice. Then to every element $a \in S$ we assign a congruence $\varphi_{a}$ as follows:

$$
\varphi_{a}=\left\{(x, y) \in S^{2} \mid x \wedge a=y \wedge a\right\} .
$$

Let $\mathscr{F}(S)$ stand for the lattice of all filters of $S$. An element $a \in S$ is called central if $[a]$ is a central element of $\mathscr{F}(S)$. Cen $(S)$ is our notation for the set of central element of $S$. If $(S, \wedge, \vee, 0,1)$ is bounded lattice, then it is easy to check that the central elements of the semilattice $(S, \wedge, 0,1)$ and of the lattice $(S, \wedge, \vee, 0,1)$ are the same. Now we have $\varphi_{c}=\theta_{\bar{c}}$ for all $c \in \operatorname{Cen}(S)$.

DEFINITION 2.4. Let $(S, \wedge, 0,1)$ be a bounded meet-semilattice.

(i) We say that an $n$-ary operation $f: S^{n} \rightarrow S$ is centre-preserving if for every $c \in \operatorname{Cen}(S),\left(x_{i}, y_{i}\right) \in \varphi_{c}, i=1, \ldots, n$, implies $\left(f\left(x_{1}, \ldots, x_{n}\right), f\left(y_{1}, \ldots, y_{n}\right)\right) \in \varphi_{c}$.

(ii) An algebra $(S, \wedge, 0,1, F)$, where $F$ is a set of operations defined on $S$, is called an algebra with a strong centre if every $f \in F$ is centre-preserving.

(iii) ( $L, \wedge, \vee, 0,1, F)$ is called an l-algebra if $(L, \wedge, 0,1, F)$ is an algebra with a strong centre and $(L, \wedge, \vee, 0,1)$ is a bounded lattice. 
The best known examples of $l$-algebras are bounded lattices, $p$-algebras, ortholattices (bounded lattices together with the orthocomplementation operation) and Heyting algebras. Implicative semilattices and $p$-semilattices (these are bounded semilattices with pseudocomplementation) are examples for algebras with a strong centre which are not $l$-algebras in general. (For details see [8].) Clearly, any $l$-algebra is congruence distributive and the factor congruences of an $l$-algebra and of its underlying lattice $L$ coincide. Thus an $l$-algebra $\mathscr{L}$ is directly indecomposable if and only if its underlying lattice $L$ is directly indecomposable. These facts together with Lemma 2.3 lead us to the following:

COROLlaRY 2.5. Let $\mathscr{L}=(L, \wedge, \vee, 0,1, F)$ be an l-algebra. Then the following assertions are satisfied:

(i) $\mathscr{L}=\prod_{i \in I} \mathscr{L}_{i}$ with $\mathscr{L}_{i}=\left(L_{i}, \wedge, \vee, 0_{i}, 1_{i}, F\right)$ if and only if $L=\prod_{i \in I} L_{i}$.

(ii) $\mathscr{L}$ is a direct product of directly indecomposable l-algebras if and only if $L$ is a direct product of directly indecomposable lattices.

A lattice $L$ with 0 element is called a pseudocomplemented lattice if for each $x \in L$ there exists an element $x^{*} \in L$ such that for any $y \in L, y \wedge x=0$ is equivalent to $y \leqq x^{*}$. If $x^{*} \vee x^{* *}=1$ holds for all $x \in L$, then $L$ is called a Stone lattice. In any Stone lattice the identity $(x \vee y)^{* *}=x^{* *} \vee y^{* *}$ is also satisfied. A complete distributive lattice $L$ is called completely Stone if $\left(\bigvee_{i \in I} x_{i}\right)^{* *}=\bigvee_{i \in I} x_{i}^{* *}$ holds for any $x_{i}, i \in I$. If $L$ is a bounded pseudocomplemented lattice, then $\left(L, \wedge, \vee, 0,1,{ }^{*}\right)$ is called a $p$-algebra.

Now let $\mathbf{A}=\prod_{i \in l} \mathbf{A}_{i}$ be a direct product of algebras $\mathbf{A}_{i}, i \in I$ and let $x_{i} \in A_{i}$ denote the $i$-th coordinate of an $x \in A$. The identical and total relations on $A$ (on $A_{i}$ ) are denoted by $\Delta_{A}, \nabla_{A}$ (by $\Delta_{i}, \nabla_{i}$ ), respectively. A congruence $\theta \in$ Con $\mathbf{A}$ is called the product of the congruences $\theta_{i} \in \operatorname{Con} \mathbf{A}_{i}$ if

$$
\theta=\left\{(a, b) \in A^{2} \mid\left(a_{i}, b_{i}\right) \in \theta_{i} \text { for each } i \in I\right\} .
$$

We write $\theta=\prod_{i \in I} \theta_{i}$ or $\theta=\theta_{1} \times \cdots \times \theta_{n}$ (when $\left.I=\{1, \ldots, n\}\right)$.

REMARK 2.6. (i) Obviously, the relations $\psi_{i} \leqq \theta_{i}, i \in I$ imply

$$
\prod_{i \in I} \psi_{i} \leqq \prod_{i \in I} \theta_{i}
$$

moreover $\prod_{i \in I} \theta_{i}=\triangle_{A}$ exactly when $\theta_{i}=\triangle_{i}$ for all $i \in I$.

(ii) For any $\theta=\prod_{i \in l} \theta_{i} \in \mathbf{C o n} \mathbf{A}$ and any $\psi=\prod_{i \in l} \psi_{i} \in$ Con $\mathbf{A}$ we have $\theta \wedge \psi=\prod_{i \in I}\left(\theta_{i} \wedge \psi_{i}\right)$ and $\theta \vee \psi \leqq \prod_{i \in I}\left(\theta_{i} \vee \psi_{i}\right)$.

Let $\operatorname{ker} \pi_{i}$ denote the kernel congruence of the natural projection $\pi_{i}: \prod_{i \in I} A_{i} \rightarrow A_{i}$, $\pi_{i}\left(x_{i}\right)=x_{i}(i \in I)$. The proof of the following lemma is implicitly contained in [1, Chapter IV, Section 11]. 
LEMMA 2.7. $\theta$ is a product congruence of the algebra $\mathbf{A}=\prod_{i \in I} \mathbf{A}_{i}$ if and only if $\theta=\bigwedge_{i \in I}\left(\theta \vee \operatorname{ker} \pi_{i}\right)$. In particular if $\mathbf{A}$ is congruence distributive and I is finite, then any congruence on $\mathbf{A}$ is a product congruence.

\section{Product decompositions of congruence distributive algebras}

In this section we deal with congruence distributive algebras. We note that the congruence lattice of such an algebra $\mathbf{A}$ is always pseudocomplemented. It is also known that in this case $\mathbf{A}=\prod_{i=1}^{n} \mathbf{A}_{i}$ implies $\operatorname{Con} \mathbf{A} \cong \prod_{i=1}^{n}$ Con $\mathbf{A}_{i}$. The main result of the present section is the following:

THEOREM 3.1. Let $\mathbf{A}=\prod_{i \in I} \mathbf{A}_{i}$ be a congruence distributive algebra and assume that all $\mathbf{A}_{i}, i \in I$ are directly indecomposable. Then the following are equivalent:

(i) A enjoys property (PCC) and Con $\mathbf{A}$ is a Stone lattice (an atomic Stone lattice).

(ii) Any $\mathbf{A}_{i}$ is finitely subdirectly irreducible (subdirectly irreducible).

First we prove the following:

LEMMA 3.2. If $\mathbf{A}=\prod_{i \in I} \mathbf{A}_{i}$ is a congruence distributive algebra, then the following statements are true:

(i) For any $\theta \in \mathrm{Con} \mathbf{A}, \theta^{*}$ is a product congruence.

(ii) If $\theta=\prod_{i \in I} \theta_{i} \in \operatorname{Con} \mathbf{A}$ with $\theta_{i} \in \operatorname{Con} \mathbf{A}_{i}$, then $\theta^{*}=\prod_{i \in I} \theta_{i}^{*}$.

(iii) For any congruence $\theta \in \operatorname{Con} \mathbf{A}, \theta \neq \triangle_{A}$ there exists a product congruence $\varphi=\prod_{i \in I} \varphi_{i}$ with $\varphi_{i} \in \operatorname{Con} \mathbf{A}_{i}$ such that $\triangle_{A}<\varphi \leqq \theta$.

PROOF. (i) Clearly, $\theta^{*} \leqq \bigwedge_{i \in I}\left(\theta^{*} \vee \operatorname{ker} \pi_{i}\right)$. On the other hand, we have

$$
\begin{aligned}
\theta \wedge\left[\bigwedge_{i \in I}\left(\theta^{*} \vee \operatorname{ker} \pi_{i}\right)\right] & =\bigwedge_{i \in I}\left[\theta \wedge\left(\theta^{*} \vee \operatorname{ker} \pi_{i}\right)\right]=\bigwedge_{i \in I}\left(\theta \wedge \operatorname{ker} \pi_{i}\right) \\
& \leqq \bigwedge_{i \in I} \operatorname{ker} \pi_{i}=\triangle_{A}
\end{aligned}
$$

whence $\bigwedge_{i \in I}\left(\theta^{*} \vee \operatorname{ker} \pi_{i}\right) \leqq \theta^{*}$. Thus we get $\theta^{*}=\bigwedge_{i \in I}\left(\theta^{*} \vee \operatorname{ker} \pi_{i}\right)$ and in view of Lemma 2.7 this means that $\theta^{*}$ is a product congruence.

(ii) We have

$$
\theta \wedge\left(\prod_{i \in I} \theta_{i}^{*}\right)=\left(\prod_{i \in I} \theta_{i}\right) \wedge\left(\prod_{i \in I} \theta_{i}^{*}\right)=\prod_{i \in I}\left(\theta_{i} \wedge \theta_{i}^{*}\right)=\prod_{i \in I} \triangle_{i}=\triangle_{A},
$$


therefore $\prod_{i \in l} \theta_{i}^{*} \leqq \theta^{*}$. Further, in view of the above (i), $\theta^{*}$ is of the form $\theta^{*}=\prod_{i \in I} \beta_{i}$ with $\beta_{i} \in \operatorname{Con} \mathbf{A}_{i}$. Thus

$$
\prod_{i \in I}\left(\theta_{i} \wedge \beta_{i}\right)=\left(\prod_{i \in I} \theta_{i}\right) \wedge\left(\prod_{i \in I} \beta_{i}\right)=\theta \wedge \theta^{*}=\triangle_{A},
$$

whence we get $\theta_{i} \wedge \beta_{i}=\Delta_{i}$ providing that $\beta_{i} \leqq \theta_{i}^{*}$ for all $i \in I$. Hence

$$
\theta^{*}=\prod_{i \in I} \beta_{i} \leqq \prod_{i \in I} \theta_{i}^{*}
$$

Summarizing, we obtain $\theta^{*}=\prod_{i \in l} \theta_{i}^{*}$.

(iii) We have $\theta \not \operatorname{ker} \pi_{i_{0}}$ for some $i_{0} \in I$, otherwise we would get

$$
\theta \leqq \bigwedge_{i \in I} \operatorname{ker} \pi_{i}=\triangle_{A}
$$

a contradiction. Since $\bigwedge_{i \in I \backslash\left\{i_{0}\right\}} \operatorname{ker} \pi_{i}$ is the complement of $\operatorname{ker} \pi_{i_{0}}$ in Con $\mathbf{A}$, we have $\theta \wedge\left(\bigwedge_{\left.i \in I \backslash i_{0}\right\}} \operatorname{ker} \pi_{i}\right) \neq \triangle_{A}$.

Set $\varphi=\theta \wedge\left(\bigwedge_{\left.i \in I \backslash i_{0}\right)} \operatorname{ker} \pi_{i}\right)$. Then $\triangle_{A}<\varphi \leqq \theta$. We claim that $\varphi$ is a product congruence.

Clearly, we have $\varphi \vee \operatorname{ker} \pi_{i}=\operatorname{ker} \pi_{i}$ for all $i \in I \backslash\left\{i_{0}\right\}$ and

$$
\begin{aligned}
\varphi \vee \operatorname{ker} \pi_{i_{0}} & =\left(\theta \vee \operatorname{ker} \pi_{i_{0}}\right) \wedge\left[\left(\bigwedge_{\left.i \in I \backslash i_{0}\right\}} \operatorname{ker} \pi_{i}\right) \vee \operatorname{ker} \pi_{i_{0}}\right] \\
& =\left(\theta \vee \operatorname{ker} \pi_{i_{0}}\right) \wedge \nabla_{A}=\theta \vee \operatorname{ker} \pi_{i_{0}} .
\end{aligned}
$$

Now, we can write:

$$
\begin{aligned}
\bigwedge_{i \in I}\left(\varphi \vee \operatorname{ker} \pi_{i}\right) & =\left(\varphi \vee \operatorname{ker} \pi_{i_{0}}\right) \wedge\left(\bigwedge_{i \in I \backslash\left\langle i_{0}\right\rfloor}\left(\varphi \vee \operatorname{ker} \pi_{i}\right)\right) \\
& =\left(\theta \vee \operatorname{ker} \pi_{i_{0}}\right) \wedge\left(\bigwedge_{i \in I \backslash\left\{i_{0}\right\}} \operatorname{ker} \pi_{i}\right) \\
& =\left[\theta \wedge\left(\bigwedge_{i \in I \backslash\left\{i_{0}\right\rfloor} \operatorname{ker} \pi_{i}\right)\right] \vee\left(\bigwedge_{i \in I} \operatorname{ker} \pi_{i}\right)=\varphi \vee \Delta_{A}=\varphi
\end{aligned}
$$

In view of Lemma $2.7 \varphi=\prod_{i \in I} \varphi_{i}$ for some $\varphi_{i} \in \operatorname{Con} \mathbf{A}_{i}$.

Proof of Theorem 3.1. (ii) implies (i). Let $\mathbf{A}=\prod_{i \in I} \mathbf{A}_{i}$ with all $\mathbf{A}_{i}$ finitely subdirectly irreducible. According to [8, Section 5, Corollary 2] Con $\mathbf{A}_{i}$ and Con $\mathbf{A}$ are Stone lattices. In order to prove that $\mathbf{A}$ obeys property (PCC) take a complemented 
congruence $\theta \in \operatorname{Con} \mathbf{A}$. Then $\theta^{*}=\bar{\theta}$ and $(\bar{\theta})^{*}=\theta$. Now, in view of Lemma 3.2 (i) there exist $\theta_{i} \in \operatorname{Con} \mathbf{A}_{i}, i \in I$ such that $\theta=\prod_{i \in I} \theta_{i}$ and Lemma 3.2 (ii) gives $\bar{\theta}=\theta^{*}=\prod_{i \in I} \theta_{i}^{*}$ and $\theta=(\bar{\theta})^{*}=\prod_{i \in I} \theta_{i}^{* *}$. As $\theta_{i}^{*} \wedge \theta_{i}^{* *}=\triangle_{i}$ and since $\mathbf{A}_{i}$ is finitely subdirectly irreducible, we get that either $\theta_{i}^{*}=\triangle_{i}$ and $\theta_{i}^{* *}=\nabla_{i}$ or $\theta_{i}^{* *}=\triangle_{i}$ and $\theta_{i}^{*}=\left(\theta_{i}^{* *}\right)^{*}=\nabla_{i}$. Take $K=\left\{i \in I \mid \theta_{i}^{*}=\triangle_{i}\right\}$. Then $I \backslash K=\left\{i \in I \mid \theta_{i}^{* *}=\triangle_{i}\right\}$ and we obtain $\mathbf{A} / \bar{\theta} \cong \prod_{i \in K} \mathbf{A}_{i}$ and $\mathbf{A} / \theta \cong \prod_{i \in I \backslash K} \mathbf{A}_{i}$.

Since

$$
\mathbf{A}=\prod_{i \in I} \mathbf{A}_{i} \cong\left(\prod_{i \in K} \mathbf{A}_{i}\right) \times\left(\prod_{i \in I \backslash K} \mathbf{A}_{i}\right) \cong \mathbf{A} / \bar{\theta} \times \mathbf{A} / \theta
$$

canonically, $\theta$ and $\bar{\theta}$ form a factor congruence pair of $A$, therefore they permute.

Finally, we show that whenever each $\mathbf{A}_{i}, i \in I$ is subdirectly irreducible, then Con $\mathbf{A}$ is an atomic lattice. Take $\theta \in \operatorname{Con} \mathbf{A}, \theta \neq \triangle_{A}$ arbitrary. In view of Lemma 3.2 (iii) there is a product congruence $\varphi=\prod_{i \in I} \varphi_{i}$ with $\varphi_{i} \in \operatorname{Con} \mathbf{A}_{i}$ and $\triangle_{A}<\varphi \leqq \theta$. Then we have $\varphi_{i_{0}} \neq \Delta_{i_{0}}$ for some $i_{0} \in I$. We define the congruence $\alpha=\prod_{i \in I} \alpha_{i}$, where $\alpha_{i_{0}}$ is the least nonzero element of Con $\mathbf{A}_{i_{0}}$ and $\alpha_{i}=\Delta_{i}$ for $i \neq i_{0}$. Clearly, $\alpha$ is an atom of Con $\mathbf{A}$ satisfying $\alpha \leqq \varphi \leqq \theta$. Thus $\operatorname{Con} \mathbf{A}$ is an atomic lattice.

(i) implies (ii). First we prove that for any $i \in I$, Con $\mathbf{A}_{i}$ is an (atomic) Stone lattice and $\mathbf{A}_{i}$ enjoys property (PCC).

For every $i \in I$, take $\mathbf{B}_{i}=\prod_{k \in I \backslash i\}} \mathbf{A}_{k}$. Then $\mathbf{A} \cong \mathbf{B}_{i} \times \mathbf{A}_{i}$ and Con $\mathbf{A} \cong$ Con $\mathbf{B}_{i} \times \operatorname{Con} \mathbf{A}_{i}$. Now Con $\mathbf{A}_{i}$ as a direct factor of the (atomic) Stone lattice Con $\mathbf{A}$ is also an (atomic) Stone lattice.

Now we prove that $\mathbf{A}_{i}$ enjoys (PCC). Take a complemented $\alpha \in \operatorname{Con} \mathbf{A}_{i}$. Let $\mathbf{B}_{i}$ denote the same algebra as above. We get $\mathbf{A} \cong \mathbf{B}_{i} \times \mathbf{A}_{i}$. Let us consider the product congruences $\varphi=\nabla_{B_{i}} \times \alpha, \bar{\varphi}=\triangle_{B_{i}} \times \bar{\alpha}$. Clearly, $\varphi$ and $\bar{\varphi}$ are complemented and by hypothesis $\varphi \circ \bar{\varphi}=\bar{\varphi} \circ \varphi$. Therefore, $\alpha \circ \bar{\alpha}=\bar{\alpha} \circ \alpha$.

Further, observe that in order to prove that $\mathbf{A}_{i}$ is finitely subdirectly irreducible, it is enough to show that $\Delta_{i}$ is a meet-irreducible element of Con $\mathbf{A}_{i}$. Assume that there are $\varphi, \theta \in \operatorname{Con} \mathbf{A}_{i}$ such that $\varphi \neq \triangle_{i}, \theta \neq \triangle_{i}$ and $\varphi \wedge \theta=\Delta_{i}$. Then we have $\theta^{*} \neq \nabla_{i}$ and $\theta^{*} \geq \varphi>\triangle_{i}$. The latter relation implies $\theta^{* *} \neq \nabla_{i}$ and we also have $\theta^{* *} \geq \theta>\triangle_{i}$. Thus we get $\theta^{*}, \theta^{* *} \notin\left\{\triangle_{i}, \nabla_{i}\right\}$. Since Con $\mathbf{A}_{i}$ is a Stone lattice, we get that $\theta^{*}$ and $\theta^{* *}$ are complements of each other. As $\mathbf{A}_{i}$ obeys (PCC), $\theta^{*}$ and $\theta^{* *}$ permute. Thus they form a factor congruence pair of $\mathbf{A}_{i}$, so we have $\mathbf{A}_{i} \cong \mathbf{A}_{i} / \theta^{*} \times \mathbf{A}_{i} / \theta^{* *}$. Since none of these two factor algebras are trivial, we get that $\mathbf{A}_{i}$ is directly decomposable, a contradiction. Therefore $\Delta_{i}$ is meet-irreducible, providing that $\mathbf{A}_{i}$ is finitely subdirectly irreducible.

Assume now that $\operatorname{Con} \mathbf{A}$ is an atomic lattice, then $\operatorname{Con} \mathbf{A}_{i}$ is also atomic (as we have already seen), and let $\alpha$ be an atom of it. Since $\Delta_{i}$ is meet-irreducible, we have $\theta \wedge \alpha=\alpha$ for all $\theta \in \operatorname{Con} \mathbf{A}_{i} \backslash\left\{\triangle_{i}\right\}$, whence we get that $\alpha \leqq \theta$ for all 
$\theta \in \operatorname{Con} \mathbf{A}_{i}, \theta \neq \triangle_{i}$. Thus any algebra $\mathbf{A}_{i}$ is subdirectly irreducible, and the proof is completed.

Since any algebra with a finite congruence lattice is a direct product of directly indecomposable algebras, the following corollary of Theorem 3.1 is immediate.

COROLlARY 3.3. A congruence distributive algebra $\mathbf{A}$ with finite $\mathrm{Con} \mathbf{A}$ is a direct product of subdirectly irreducible algebras if and only if $\mathbf{A}$ enjoys (PCC) and Con A is a Stone lattice.

The following consequence of Theorem 3.1 can be considered as a completion of [8, Theorem 1.2].

PROPOSITION 3.4. Let $\mathbf{A}$ be a congruence distributive algebra with a strong centre. Then the following assertions are equivalent:

(i) A enjoys (PCC) and Con $\mathbf{A}$ is a completely Stonean (atomic) lattice.

(ii) $\mathbf{A}$ is a finite direct product of finitely subdirectly irreducible (subdirectly irreducible) algebras.

Proof. Applying Theorem 1.2 we get that (i) implies (ii).

Now we prove that (ii) implies (i). In view of Theorem 3.1 the assumption of (ii) implies that $\mathbf{A}$ obeys property (PCC) (and Con $\mathbf{A}$ is a Stone lattice). Applying again Theorem 1.2 we get that $\mathbf{C o n} \mathbf{A}$ is a completely Stonean (atomic) lattice.

An other remarkable result of [8] is the following (see [8, Theorem 6 (iii)]): 'Let $\mathbf{A}$ be a congruence distributive algebra with a strong centre and let $\mathbf{A}$ enjoy the property (PCC). Then Con $\mathbf{A}$ is a Boolean lattice if and only if $\mathbf{A}$ is a finite direct product of simple algebras.'

By using this result and Theorem 3.1 we prove:

PROPOSITION 3.5. Let $\mathbf{A}$ be a congruence distributive algebra with a strong centre. Then $\mathbf{A}$ is a finite direct product of simple algebras if and only if $\mathbf{A}$ is congruence permutable and Con $\mathbf{A}$ is a Boolean lattice.

PROOF. In view of the above cited theorem of [8] our proof is quite similar to the proof of Proposition 3.4. In addition we have only to prove that a congruence distributive algebra $\mathbf{A}=\prod_{i=1}^{n} \mathbf{A}_{i}$ with all $\mathbf{A}_{i}$ simple is congruence permutable. Since now any $\theta \in \operatorname{Con} \mathbf{A}$ is of the form $\theta=\prod_{i=1}^{n} \theta_{i} \in \operatorname{Con} \mathbf{A}$ with $\theta_{i} \in\left\{\triangle_{i}, \nabla_{i}\right\}$, this assertion is obvious.

REMARK 3.6. As any $l$-algebra is congruence distributive with a strong centre, Propositions 3.4 and 3.5 also apply to the case of $l$-algebras. We note that Proposition 3.5 generalizes Dilworth's result from [2]. 
An element $p \in L \backslash\{0\}$ of a (complete) lattice $L$ is called completely join-irreducible if for any system of elements $x_{i} \in L, i \in I$ the equality $p=\bigvee\left\{x_{i} \mid i \in I\right\}$ implies $p=$ $x_{i_{0}}$ for some $i_{0} \in I$. If any nonzero element of $L$ is a join of completely join-irreducible elements, then $L$ is called a $C J$-generated lattice. In view of Libkin's result [9], any CJ-generated algebraic lattice is a direct product of directly indecomposable lattices. Therefore, by applying Theorem 3.1 we can add to Theorem 1.1 the following:

COROLLARY 3.7. Let $L$ be a CJ-generated algebraic lattice. Then $L$ is a direct product of finitely subdirectly irreducible lattices if and only if $L$ enjoys property (PCC) and $\operatorname{Con} L$ is a Stone lattice.

\section{Lattices which are direct products of directly indecomposable lattices}

The difficulty to apply Theorem 3.1 to obtain product decompositions of $l$-algebras (where the decomposition may contain an infinite number of factors) is that we do not even know under what conditions an arbitrary $l$-algebra can be written as a direct product of directly indecomposable $l$-algebras. In view of Corollary 2.5 such a direct decomposition of an $l$-algebra exists if and only if the underlying lattice is a direct product of directly indecomposable lattices. Therefore in this section we shall establish a necessary and sufficient condition (Theorem 4.2) for the existence of the above mentioned direct decomposition of bounded lattices.

The following notion will play an important role in our investigation.

DEFINITION 4.1. A bounded lattice $L$ is called weakly central-complete if for any set $\left\{a_{k} \in \operatorname{Cen}(L) \mid k \in K\right\}$ of distinct atoms of $\operatorname{Cen}(L)$ and for any set $\left\{x_{k} \in L \mid x_{k} \leqq a_{k}\right.$, $k \in K$ \} of elements the join $\bigvee_{k \in K} x_{k}$ exists in $L$.

Obviously, any complete lattice and any bounded lattice whose center contains a finite number of atoms is weakly central-complete. The following theorem clarifies the role of the above notion.

THEOREM 4.2. Let $L$ be a bounded nontrivial lattice. Then the following assertions are equivalent:

(i) $L \cong \prod_{i \in I} L_{i}$ with directly indecomposable (nontrivial) $L_{i}$ 's.

(ii) $\operatorname{Cen}(L)$ is an atomic lattice, $L$ is weakly central-complete and for any set of elements $c_{j} \in \operatorname{Cen}(L), j \in J$ there is a $u \in \operatorname{Cen}(L)$ such that $\bigwedge_{j \in J} \theta_{c_{j}}=\theta_{u}$.

ProOF. (i) implies (ii). Clearly, we can restrict our consideration to the case $L=\prod_{i \in I} L_{i}$. For each $M \subseteq I$ we define the elements $c^{M} \in \prod_{i \in I} L_{i}$ by $\left(c^{M}\right)_{i}=1_{i}$ for all $i \in M$, otherwise $\left(c^{M}\right)_{i}=0_{i}$. It can be easily seen that $c^{M} \in \operatorname{Cen}(L)$ and 
$L_{i} \cong\left(c^{(i)}\right]$, providing that every sublattice $\left(c^{(i)}\right]$ is directly indecomposable (see also Lemma 2.3). It is also easy to see that any $c^{\{i\}}$ is an atom of Cen $(L)$. (Indeed, if an element $c \in \operatorname{Cen}(L)$ with $0<c<c^{(i)}$ would exist, then $c$ and $\bar{c} \wedge c^{\{i\}}$ would form a complemented pair of central elements of the sublattice $\left(c^{(i)}\right]$.)

Take any $a \in \operatorname{Cen}(L), a \neq 0$. We claim that $a=c^{M}$ for some $M \leqq I$. As $c^{(i)}$ is an atom of $\operatorname{Cen}(L)$ and $a \wedge c^{(i)} \in \operatorname{Cen}(L)$, we get for each $i \in I$ either $a \wedge c^{\{i\}}=0$ or $a \wedge c^{\{i\}}=c^{\{i\}}$, that is, $a_{i}=0_{i}$ or $a_{i}=1_{i}$. Then $a=c^{M}$, where $M=\left\{i \in I \mid a_{i}=1\right\}$. Since any nonzero element of $\operatorname{Cen}(L)$ is of the form $c^{M}$ with $M \neq \emptyset$ and $c^{\left.\mid i_{0}\right\}} \leqq c^{M}$ for any $i_{0} \in M$, we deduce that $\left\{c^{(i)} \mid i \in I\right\}$ is the set of all atoms of $\operatorname{Cen}(L)$ and $\operatorname{Cen}(L)$ is atomic.

Now take the elements $c_{j} \in \operatorname{Cen}(L), j \in J$; then $c_{j}=c^{M_{j}}$ for some $M_{j} \subseteq I$. It is easy to check that $\theta_{c^{M}}=\left\{(x, y) \in L^{2} \mid(x \vee y)_{i}=(x \wedge y)_{i}\right.$ for all $\left.i \notin M\right\}$. In consequence $\theta_{c^{M}}=\left\{(x, y) \in L^{2} \mid x_{i}=y_{i}\right.$ for all $\left.i \notin M\right\}$. We claim that $\bigwedge_{j \in J} \theta_{c_{j}}=\theta_{u}$, where $u=c^{\bigcap_{j \in J} M_{j}}$. Indeed,

$$
\begin{aligned}
\bigwedge_{j \in J} \theta_{c_{j}} & =\bigcap_{j \in J}\left\{(x, y) \in L^{2} \mid x_{i}=y_{i} \text { for each } i \in I \backslash M_{j}\right\} \\
& =\left\{(x, y) \in L^{2} \mid x_{i}=y_{i} \text { for all } i \in \bigcup_{j \in J}\left(I \backslash M_{j}\right)\right\} \\
& =\left\{(x, y) \in L^{2} \mid x_{i}=y_{i} \text { for all } i \in I \backslash\left(\bigcap_{\in \in J} M_{j}\right)\right\}=\theta_{u} .
\end{aligned}
$$

Finally, a nonempty set of distinct atoms of $\operatorname{Cen}(L)$ can be written as $A=\left\{c^{|k|} \mid k \in K\right\}$, where $\emptyset \neq K \subseteq I$. Take any set $\left\{x_{k} \in L \mid k \in K\right\}$ with $x_{k} \leqq c^{[k]}$. Now we have:

$$
\left(x_{k}\right)_{k} \leqq 1_{k} \quad \text { and } \quad\left(x_{k}\right)_{i} \leqq\left(c^{|k\rangle}\right)_{i}=0_{i} \quad \text { for all } i \neq k .
$$

Define $x^{\Pi} \in L$ as follows: $\left(x^{\Pi}\right)_{i}=0_{i}$ for all $i \in I \backslash K$ and $\left(x^{\Pi}\right)_{i}=\left(x_{i}\right)_{i}$ for all $i \in K$. Then, in view of $(*)$, we have $\left(x_{k}\right)_{i} \leqq\left(x^{\Pi}\right)_{i}$ for all $i \in I$ and this gives $x_{k} \leqq x^{\Pi}$ for all $k \in K$. Let $y \in L$ be an arbitrary upper bound of $\left\{x_{k} \mid k \in K\right\}$. Then $\left(x_{k}\right)_{i} \leqq y_{i}$ for all $i \in I$ and $k \in K$, whence we get $\left(x_{k}\right)_{k} \leqq y_{k}$ for all $k \in K$. Now we have $x^{\Pi} \leqq y$, by the definition of $x^{\Pi}$. Therefore $x^{\Pi}$ is the least upper bound of $\left\{x_{k} \in L \mid k \in K\right\}$ in $L$, that is, $x^{\Pi}=\bigvee_{k \in K} x_{k}$. Thus $L$ is weakly central-complete.

(ii) implies (i). First we show that $\operatorname{Cen}(L)$ is a complete sublattice of $L$. Take $c_{k} \in \operatorname{Cen}(L), k \in K$; then, by our assumption, $\bigwedge_{k \in K} \theta_{c_{k}}=\theta_{u}$ for some $u \in \operatorname{Cen}(L)$. Since $\theta_{u} \leqq \theta_{c_{k}}$ implies $u \leqq c_{k}, k \in K$, we get that $u$ is a lower bound of the set $\left\{c_{k} \mid k \in K\right\}$. On the other hand, for any lower bound $l \in L$ of $\left\{c_{k} \mid k \in K\right\}$ we have $(0, l) \in \theta_{c_{k}}, k \in K$. Thus $(0, l) \in \bigwedge_{k \in K} \theta_{c_{k}}=\theta_{u}$, whence we get $l \leqq u$ proving $u=\bigwedge_{k \in K} c_{k}$. Therefore $\bigwedge_{k \in K} c_{k}$ exists in $L$ and $\bigwedge_{k \in K} c_{k}=u \in \operatorname{Cen}(L)$, moreover we obtained that $\bigwedge_{k \in K} \theta_{c_{k}}=\theta_{\bigwedge_{k \in K} c_{k}}$. 
Now take $v=\bigwedge_{k \in K} \overline{c_{k}}$. Then $v \in \operatorname{Cen}(L)$ and so $\bar{v} \geqq c_{k}, k \in K$, thus $\bar{v}$ is a upper bound for $\left\{c_{k} \mid k \in K\right\}$. Let $a \in L$ with $a \geqq c_{k}, k \in K$, then we have $(a, 1) \in \bigwedge_{k \in K} \theta_{\overline{c_{k}}}=\theta_{v}$, according to the definition of congruences $\theta_{\overline{\bar{k}_{k}}}$. So we get $a \vee v=1$, implying $\bar{v}=(a \vee v) \wedge \bar{v}=a \wedge \bar{v}$. Hence $\bar{v} \leqq a$. Therefore $\bigvee_{k \in K} c_{k}$ exists in $L$ and $\bigvee_{k \in K} c_{k}=\bar{v} \in \operatorname{Cen}(L)$.

Since $\operatorname{Cen}(L)$ is an atomic complete Boolean lattice, it is atomistic and infinitely distributive too. (Even more it is completely distributive.) Let $\left\{a_{i} \mid i \in I\right\}$ be the set of all atoms of $\operatorname{Cen}(L)$. Then $\bigvee_{i \in I} a_{i}=1$ and we prove that $L \cong \prod_{i \in I}\left(a_{k}\right]$.

Let us define the map $f: L \rightarrow \prod_{i \in I}\left(a_{i}\right]$, by setting $(f(x))_{i}=x \wedge a_{i}, i \in I$ for all $x \in L$ (where $x_{i}$ stands for the $i$-th coordinate of an $x \in \prod_{i \in I}\left(a_{i}\right]$ ). It is not hard to check that $f$ is a homomorphism.

In order to prove that $f$ is one to one take $x, y \in L$ with $f(x)=f(y)$; then

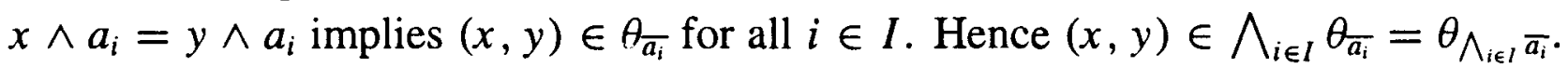
Since $\operatorname{Cen}(L)$ is an infinitely distributive Boolean lattice $\bigwedge_{i \in l} \overline{a_{i}} \in \operatorname{Cen}(L)$ is the complement of $\bigvee_{i \in I} a_{i}=1$. Thus we have $\bigwedge_{i \in I} \overline{a_{i}}=0$, and this implies $(x, y) \in$ $\theta_{0}=\triangle_{L}$. Hence we get $x=y$.

To prove that $f$ is onto, take a $y=\left(y_{i}\right)_{i \in I} \in \prod_{i \in I}\left(a_{i}\right]$. Since $y_{i} \leqq a_{i}, i \in I$ and since $L$ is weakly central-complete, the join $z=\bigvee_{i \in I} y_{i}$ exists in $L$. We claim that $f(z)=y$.

Indeed, we have $y_{k} \leqq\left(\bigvee_{i \in I} y_{i}\right) \wedge a_{k}=z \wedge a_{k}$ for all $k \in I$. On the other hand we can write:

$$
z \wedge a_{k}=\left(\bigvee_{i \in I} y_{i}\right) \wedge a_{k} \leqq\left[y_{k} \vee\left(\bigvee_{i \in I \backslash k\}} a_{i}\right)\right] \wedge a_{k}=\left(y_{k} \wedge a_{k}\right) \vee\left[\left(\bigvee_{i \in I \backslash k\}} a_{i}\right) \wedge a_{k}\right]
$$

Since $\operatorname{Cen}(L)$ is infinitely distributive, we have

$$
\left(\bigvee_{i \in I \backslash\{k\}} a_{i}\right) \wedge a_{k}=\bigvee_{i \in I \backslash\langle k\rfloor}\left(a_{i} \wedge a_{k}\right)=0
$$

Now $y_{k} \leqq a_{k}$ implies that $z \wedge a_{k} \leqq y_{k}$. Thus $z \wedge a_{k}=y_{k}$ for all $k \in I$, whence we get $f(z)=\left(z \wedge a_{k}\right)_{k \in I}=\left(y_{k}\right)_{k \in I}=y$, providing that $f$ is onto. Hence the map $f$ is an isomorphism and this completes the proof.

\section{The proof of main theorem}

To present the proof of Theorem 1.3 we need some essential remarks on the Boolean part of a pseudocomplemented lattice.

If $L$ is a pseudocomplemented lattice, then the set $\left\{x \in L \mid x^{* *}=x\right\}$ is called the Boolean part of $L$ and it is denoted by $\mathrm{B}(L)$. Since the identity $\bigwedge_{i \in I} x_{i}^{* *}=$ 
$\left(\bigwedge_{i \in I} x_{i}^{* *}\right)^{* *}$ is satisfied in any complete pseudocomplemented lattice, $\mathrm{B}(L)$ is a complete $\wedge$-subsemilattice of any complete $L$. If $L$ is a Stone lattice, then $\mathrm{B}(L)$ is a Boolean sublattice of $L$ (see [4]). (We note that $\mathrm{B}(L)$ is not necessarily a complete sublattice of $L$, since for $x_{i} \in \mathrm{B}(L), i \in I$ the relation $\bigvee_{i \in I} x_{i} \in \mathrm{B}(L)$ is not true in general.) Now we formulate:

LEMMA 5.1. Let $\mathbf{A}$ be a congruence distributive algebra, then

(i) If $\mathrm{Con} \mathbf{A}$ is an atomic lattice, then $\mathrm{B}(\mathrm{Con} \mathbf{A})$ is also atomic.

(ii) If $\mathbf{A}$ enjoys property (PCC) and $\mathrm{Con} \mathbf{A}$ is a Stone lattice, then $\mathrm{B}(\mathrm{Con} \mathbf{A})$ coincides with the set of all factor congruences of $\mathbf{A}$.

Proof. (i) Let $\theta \in$ Con $\mathbf{A}$ be an atom. We claim that $\theta^{* *}$ is an atom of $\mathrm{B}(\operatorname{Con} \mathbf{A})$. Really, let $\alpha<\beta=\beta^{* *} \leqq \theta^{* *}$ and $\alpha$ is an atom of $\mathrm{B}(\mathrm{Con} \mathbf{A}), \alpha \neq \theta$ implies $\alpha \wedge \theta=\Delta$. Therefore, $\Delta=\Delta^{* *}=(\alpha \wedge \theta)^{* *}=\alpha^{* *} \wedge \theta^{* *}=\alpha^{* *}$, a contradiction. Now $\alpha=\theta$ and it follows $\beta^{* *}=\theta^{* *}$, proving that $\theta^{* *}$ is an atom of $\mathrm{B}(\operatorname{Con} \mathbf{A})$.

Now, take any $\varphi \in \mathrm{B}(\operatorname{Con} \mathbf{A})$. As Con $\mathbf{A}$ is atomic, there exists an atom $\theta \in \operatorname{Con} \mathbf{A}$ such that $\theta \leqq \varphi$, whence we get $\theta^{* *} \leqq \varphi^{* *}=\varphi$.

(ii) Since $\operatorname{Con} \mathbf{A}$ is a pseudocomplemented distributive lattice, for any factor congruence $\theta \in \mathrm{Con} \mathbf{A}$ we have $\theta^{*}=\bar{\theta}$ and $(\bar{\theta})^{*}=\overline{(\bar{\theta})}=\theta$, that is $\theta^{* *}=\theta$. Hence $\theta \in \mathbf{B}(\operatorname{Con} \mathbf{A})$.

Conversely, take any $\theta \in \mathrm{B}(\operatorname{Con} \mathbf{A})$. As $\operatorname{Con} \mathbf{A}$ is a Stone lattice and $\theta=\theta^{* *}$, we can write: $\theta \vee \theta^{*}=\theta^{* *} \vee \theta^{*}=\nabla$, therefore $\theta$ and $\theta^{*}$ are the complements of each other. Now property (PCC) implies $\theta \circ \theta^{*}=\theta^{*} \circ \theta$, providing that $\theta$ is a factor congruence of $\mathbf{A}$.

We note that the above result (ii) is contained in [8] in an implicit form.

ProOF OF THEOREM 1.3. Let $\mathscr{L}=(L, \wedge, \vee, 0,1, F)$ be an $l$-algebra such that $\mathscr{L}=\prod_{i \in I} \mathscr{L}_{i}$ with $\mathscr{L}_{i}=\left(L_{i}, \wedge, \vee, 0,1, F\right)$, all $\mathscr{L}_{i}, i \in I$ being finitely subdirectly irreducible. Since finitely subdirectly irreducible algebras are directly indecomposable and any $l$-algebra is congruence distributive, we can apply Theorem 3.1 and this gives that $\mathscr{L}$ enjoys property (PCC) and Con $\mathscr{L}$ is a Stone lattice. Moreover, if all $\mathscr{L}_{i}$ are subdirectly irreducible then Theorem 3.1 gives in addition that Con $\mathscr{L}$ is atomic. By Corollary 2.5 we have $L=\prod_{i \in I} L_{i}$ with directly indecomposable $L_{i}$ 's. Now Theorem 4.2 implies that $L$ is a weakly central-complete lattice with an atomic center. Thus we have verified the 'only if' part for both of assertions (1) and (2).

Now we prove the converse implications.

Let $\mathscr{L}=(L, \wedge, \vee, 0,1, F)$ be an $l$-algebra satisfying property (PCC) and such that $L$ is weakly central-complete and $\operatorname{Con} \mathscr{L}$ is a Stone lattice. Take $c_{j} \in \operatorname{Cen}(L)$, $j \in J$; then the congruences $\theta_{c_{j}}, j \in J$ are factor congruences of $L$ and thereby of the whole algebra $\mathscr{L}$. Thus, in view of Lemma 5.1 (ii), we have $\theta_{c_{j}} \in \mathrm{B}(\operatorname{Con} \mathscr{L})$ 
for all $j \in J$. Since $\mathrm{B}(\operatorname{Con} \mathscr{L})$ is a complete $\wedge$-subsemilattice of Con $\mathscr{L}$, we get $\bigwedge_{j \in J} \theta_{c_{j}} \in \mathrm{B}($ Con $\mathscr{L})$. Using Lemma 5.1 (ii) again we obtain that $\bigwedge_{j \in J} \theta_{c_{j}}$ is a factor congruence of the algebra $\mathscr{L}$, and so it is a factor congruence of the lattice $L$. Therefore, there is an element $u \in \operatorname{Cen}(L)$ such that

$$
\bigwedge_{j \in J} \theta_{c_{j}}=\theta_{u}
$$

Let us observe also, that the map $\psi: \theta \mapsto \theta_{c}$ in this case is a $\operatorname{Cen}(L) \rightarrow \mathrm{B}(\operatorname{Con} \mathscr{L})$ isomorphism. Indeed, in view of Remark 2.2 and Corollary $2.5 \psi$ is an injective homomorphism and the above argument gives that any $\theta \in \mathrm{B}(\operatorname{Con} \mathscr{L})$ is of the form $\theta=\theta_{c}, c \in \operatorname{Cen}(L)$, that is, $\psi$ is onto.

Case (1). Since $\operatorname{Cen}(L)$ is atomic and $L$ is weakly central-complete and satisfies (**), Theorem 4.2 implies that $L \cong \prod_{i \in I} L_{i}$ with all $L_{i}, i \in I$ directly indecomposable. Therefore, Corollary 2.5 gives that $\mathscr{L} \cong \prod_{i \in I} \mathscr{L}_{i}$, where all $\mathscr{L}_{i}=\left(L_{i}, \wedge, \vee, 0_{i}, 1_{i}, F\right)$ are directly indecomposable $l$-algebras. Since the algebra $\prod_{i \in I} \mathscr{L}_{i}$ enjoys (PCC) and its congruence lattice is Stonean, applying Theorem 3.1 we get that all $\mathscr{L}_{i}, i \in I$ are finitely subdirectly irreducible, completing the proof of (1).

Case (2). Now $L$ is weakly central-complete and Con $\mathscr{L}$ is atomic, moreover we already have shown that $L$ satisfies the property (**). Further, in view of Lemma 5.1 (i) the lattice $\mathrm{B}(\operatorname{Con} \mathscr{L})$ is atomic. As we have already seen that $\operatorname{Cen}(L) \cong \mathrm{B}(\operatorname{Con} \mathscr{L})$, we obtain that $\operatorname{Cen}(L)$ is also atomic.

Using the facts that $\operatorname{Cen}(L)$ is atomic and $L$ is weakly central-complete and that $L$ satisfies (**) we can repeat the argument in the 'if' part of the proof of assertion (1) providing that $\mathscr{L}$ is a direct product of directly indecomposable $l$-algebras. Since $\mathscr{L}$ obeys (PCC) and Con $\mathscr{L}$ is an atomic Stone lattice, Theorem 3.1 implies that the above direct factors of $\mathscr{L}$ are subdirectly irreducible $l$-algebras. This completes the proof.

REMARK 5.2. (i) Applying Proposition 3.4 to $l$-algebras we get that the product decomposition given by Theorem 1.3 consists of finite factors if and only if $\mathscr{L}$ admits (PCC) and Con $\mathscr{L}$ is completely Stonean (and atomic in case (2)).

(ii) We note that in [8] it is also proved (see [8, Theorem 6 (i)]) that $\operatorname{Cen}(\mathscr{L})$ is finite whenever $\mathscr{L}$ obeys (PCC) and Con $\mathscr{L}$ is completely Stonean. Since $\operatorname{Cen}(L)=$ $\operatorname{Cen}(\mathscr{L})$, in this case $\operatorname{Cen}(L)$ is atomic and $L$ is weakly central-complete.

\section{Applications to certain classes of $l$-algebras and lattices}

In [8] it was established that double p-algebras, ortholattices and Heyting algebras enjoy property (PCC). (A double p-algebra is an $l$-algebra $\left(L, \wedge, \vee,{ }^{*},{ }^{+}\right)$, where 
$\left(L, \wedge, \vee,{ }^{*}\right)$ is a $p$-algebra and $\left(L, \vee, \wedge,^{+}\right)$is a dual $p$-algebra.) Combining the above observations and Theorem 1.3 we obtain

COROLlaRY 6.1. Let $\mathscr{L}$ be any double p-algebra (ortholattice, Heyting algebra). Then the following are equivalent:

(i) Con $\mathscr{L}$ is Stonean, the underlying lattice $L$ is weakly-central complete and $\operatorname{Cen}(L)(\operatorname{Con} L)$ is atomic.

(ii) $\mathscr{L}$ is a direct product of finitely subdirectly irreducible (subdirectly irreducible) algebras.

Since any bounded lattice is an $l$-algebra, Theorem 3.1 also can be applied to bounded lattices. In [11] the present author proved the equivalence of the following conditions:

(i) $L$ enjoys property (PCC) and Con $L$ is a Stone lattice,

(ii) For any $\theta \in \operatorname{Con} L$, there exists a $c \in \operatorname{Cen}(L)$ such that $\theta^{*}=\theta_{c}$.

Thus we obtain the following

COROLlARY 6.2. Let $L$ be a bounded lattice. Then the following assertions are equivalent:

(i) $L$ is a direct product of finitely subdirectly irreducible (subdirectly irreducible) lattices.

(ii) $L$ is weakly central-complete, enjoys property ( $\mathrm{PCC}), \operatorname{Con} L$ is a Stone lattice and $\operatorname{Cen}(L)(\operatorname{Con} L)$ is atomic.

(iii) $L$ is weakly central-complete, for any $\theta \in \operatorname{Con} L$ there is a $c \in \operatorname{Cen}(L)$ such that $\theta^{*}=\theta_{c}$ and $\operatorname{Cen}(L)(\operatorname{Con} L)$ is atomic.

COROLLARY 6.3. A Boolean lattice $L$ is isomorphic to a power set lattice $\mathscr{P}(I)$ if and only if $\mathrm{Con} L$ is an atomic Stone lattice.

Proof. Obviously, any Boolean lattice obeys (PCC) and by [3] Con $L$ is a Stone lattice if and only if $L$ is complete. (See also [6] and [7].) Since a subdirectly irreducible Boolean lattice is isomorphic to 2 (the two element chain), $L$ is a direct product of subdirectly irreducible lattices exactly when $L \cong 2^{I}$ for some $I \neq \emptyset$. As $2^{l} \cong \mathscr{P}(I)$, our result can be derived from Corollary 6.2.

\section{Applications to certain classes of complete lattices}

In [6] Janowitz proved that any complemented lattice enjoys property (PCC) and exhibited several classes of lattices with Stonean congruence lattice. Here we mention some examples: 
- uniquely complemented complete lattices,

- weakly modular sectionally complemented complete lattices.

Since any complete lattice is bounded and weakly central-complete, Corollary 6.2 implies

COROLLARY 7.1. Let $L$ be a lattice from one of the above classes. Then $L$ is a direct product of finitely subdirectly irreducible (subdirectly irreducible) lattices if and only if $\operatorname{Cen}(L)(\operatorname{Con} L)$ is atomic.

A lattice $L$ with 0 is called section semicomplemented (SeSC) if for every $a, b \in L$, $a<b$ there is an element $0<u \leqq b$ such that $a \wedge u=0 . L$ is dually section complemented (DSeSC) if its dual $L^{(d)}$ is section semicomplemented (see [10]). If $L$ is a complete lattice then for any $\varphi \in \operatorname{Con} L$ we define $w(\varphi) \in L$ as the supremum of its kernel: $w(\varphi)=\bigvee\{x \in L \mid(0, x) \in \varphi\}$. Janowitz proved that whenever $L$ is both $\operatorname{SeSC}$ and DSeSC then we have $w(\varphi) \in \operatorname{Cen}(L)$ for any $\varphi \in \operatorname{Con} L$ (see [5, Theorem 4.17 (ii)]). He also proved that the congruence lattice of a lattice which is both SeSC and DSeSC is a Stone lattice. Now we can proceed further:

PROPOSITION 7.2. Let L be a complete lattice which is both SeSC and DSeSC. Then $L$ is a direct product of finitely subdirectly irreducible (subdirectly irreducible) lattices if and only if $\operatorname{Cen}(L)(\operatorname{Con} L)$ is atomic.

PROOF. Take any $\varphi \in \operatorname{Con} L$. We only have to prove $\varphi^{*}=\theta_{w\left(\varphi^{*}\right)}$, and then the statement of the proposition follows by applying Corollary 6.2.

Let us show first that $\varphi^{*} \leqq \theta_{w\left(\varphi^{*}\right)}$. Clearly, $\theta_{w\left(\varphi^{*}\right)} \vee \theta_{\overline{w\left(\varphi^{*}\right)}}=\nabla_{L}$. As we have $\varphi^{*}=\left(\varphi^{*} \wedge \theta_{w\left(\varphi^{*}\right)}\right) \vee\left(\varphi^{*} \wedge \theta \overline{w\left(\varphi^{*}\right)}\right)$, the relation $\varphi^{*} \wedge \theta_{\overline{w\left(\varphi^{*}\right)}}=\Delta_{L}$ implies $\varphi^{*} \leqq \theta_{w\left(\varphi^{*}\right)}$. Thus it is sufficient to verify that $\varphi^{*} \wedge \theta \overline{w_{\left(\varphi^{*}\right)}}=\Delta_{L}$.

Contrary, suppose that there exist $a, b \in L, a<b$ such that $(a, b) \in \varphi^{*} \wedge \theta \overline{w\left(\varphi^{*}\right)}$. Since $L$ is section semicomplemented, there is a $u \in L, 0<u \leqq b$ such that $a \wedge u=0$. Then $(0, u) \in \varphi^{*} \wedge \theta_{\overline{w\left(\varphi^{*}\right)}}$, so we get $(0, u) \in \varphi^{*}$ and $u \leqq \overline{w\left(\varphi^{*}\right)}$. Now $(0, u) \in \varphi^{*}$ gives $u \leqq w\left(\varphi^{*}\right)$, whence we obtain that $u \leqq w\left(\varphi^{*}\right) \wedge \overline{w\left(\varphi^{*}\right)}=0$, a contradiction.

Further, we have to show that $\theta_{w\left(\varphi^{*}\right)} \leqq \varphi^{*}$. If it is not the case, then there exist $c, d \in$ $L, c<d$ such that $(c, d) \in \theta_{w\left(\varphi^{*}\right)} \wedge \varphi$. As $L$ is dually section semicomplemented, there is a $v \in L, c \leqq v<1$ such that $d \vee v=1$. Then $(v, 1) \in \theta_{w\left(\varphi^{*}\right)} \wedge \varphi$ and this means that $v \vee w\left(\varphi^{*}\right)=1$ and $(v, 1) \in \varphi$. Observe, that for every $x \in L$ with $(0, x) \in \varphi^{*}$ we have now $(v \wedge x, x) \in \varphi \wedge \varphi^{*}=\triangle_{L}$, whence we get that $x=v \wedge x$, that is, $x \leqq v$. Therefore we obtain $w\left(\varphi^{*}\right) \leqq v$, implying that $v \vee w\left(\theta^{*}\right)=v \neq 1$, a contradiction.

Hence we conclude that $\varphi^{*}=\theta_{w\left(\varphi^{*}\right)}$, and this completes the proof. 


\section{Acknowledgment}

The author wishes to express his gratitude to professor T. Katriňák, who drew his attention to this field of research and whose results had a decisive role in preparing this paper. The author would also like to thank professors E. T. Schmidt and J. Szigeti for their several suggestions and support.

\section{References}

[1] S. Burris and H. P. Sankappanavar, A course in universal algebra (Springer, New York, 1981).

[2] R. P. Dilworth, 'The structure of relatively complemented lattices', Ann. of Math. 51 (1950), 348-359.

[3] O. Frink, 'Pseudo-complements in semi-lattices', Duke Math. J. 29 (1962), 505-514.

[4] G. Grätzer, General lattice theory (Academic Press, New York, 1978).

[5] M. F. Janowitz, 'Section semicomplemented lattices', Math. Z. 108 (1968), 63-76.

[6] - 'Complemented congruences on complemented lattices', Pacific J. Math. 73 (1977), 87-90.

[7] T. Katriňák, 'Notes on Stone lattices II', Mat. Fyz. Časop. SAV 17 (1967), 20-37 (In Russian).

[8] T. Katriňák and S. El-Assar, 'Algebras with Boolean and Stonean congruence lattices', Acta Math. Hungar. 48 (1986), 301-316.

[9] L. Libkin, 'Direct decomposition of atomistic algebraic lattices', Algebra Universalis 33 (1995), 127-135.

[10] F. Maeda and S. Maeda, Theory of symmetric lattices (Springer, Berlin, 1970).

[11] S. Radeleczki, 'Maeda-type decomposition of CJ-generated algebraic lattices', South East Asian Bull. Math. 25 (2001), 503-513.

Institute of Mathematics

University of Miskolc-Egyetemváros

3515 Miskolc

Hungary

e-mail: matradi@gold.uni-miskolc.hu 